\title{
Coagulación intravascular diseminada: Una revisión de tema
}

\author{
Disseminated intravascular coagulation: A review of the topic
}

Alejandro Hernández-Martínez1 orcid.org/0000-0001-6577-9666

Lina María Martínez-Sánchez1* orcid.org/0000-0002-9555-0843

1 Grupo de Investigación Medicina Interna, Facultad de Medicina, Universidad Pontificia Bolivariana. Medellín, Colombia

Fecha de recepción: Septiembre 25-2017 Fecha de revisión: Junio 19 - $2018 \quad$ Fecha de aceptación: Agosto 14 - 2018

Hernández-Martínez A, Martínez-Sánchez LM. Coagulación intravascular diseminada: una revisión de tema. Univ. Salud. 2018;20(3):283-291. DOI: http://dx.doi.org/10.22267/rus.182003.132

\begin{abstract}
Resumen
Introducción: La Coagulación Intravascular Diseminada, es un síndrome secundario a patologías subyacentes, donde la activación localizada de la coagulación y la respuesta inflamatoria generalizada, pueden llevar a daños tisulares y microvasculares. Se ha reportado una prevalencia del 10,8\% en varias unidades de cuidados intensivos de Colombia; su presentación en el contexto de sepsis es diferente según el tipo de infección. Objetivo: Realizar una revisión de la literatura de los conceptos más destacados de la Coagulación Intravascular Diseminada. Materiales y métodos: Se realizó una revisión por medio de la búsqueda de artículos originales, revisiones sistemáticas y narrativas, en las bases de datos PubMed y ScienceDirect y en el buscador Google Académico; se seleccionaron 80 artículos, de los cuales se incluyeron 51. Se tuvo en cuenta publicaciones en español, inglés y francés, con fecha de publicación menor o igual a 5 años. Resultados: Se realizó la descripción sobre tratamiento, etiología, presentación clínica y diagnóstico de la Coagulación Intravascular Diseminada, haciendo especial énfasis en los estudios sobre marcadores moleculares y nuevas alternativas terapéuticas. Conclusión: La Coagulación Intravascular Diseminada es una complicación que contribuye a aumentar la morbilidad y la mortalidad, cuyo pronto diagnóstico y tratamiento aportan significativamente a una mejor evolución clínica.
\end{abstract}

Palabras clave: Coagulación intravascular diseminada; sepsis; anticoagulantes. (Fuente: DeCS, Bireme).

\begin{abstract}
Introduction: Disseminated intravascular coagulation is a secondary syndrome to underlying pathologies, where localized coagulation activation and generalized inflammatory response can lead to tissue and microvascular damage. A prevalence of $10.8 \%$ has been reported in several intensive care units in Colombia. Its presentation in the context of sepsis is different depending on the type of infection. Objective: To conduct a review of literature of the most outstanding concepts of disseminated intravascular coagulation. Materials and methods: A review was made by means of the search of original articles, systematic and narrative reviews, in the PubMed and ScienceDirect databases and in the Google Scholar search engine; 80 articles were selected, of which 51 were included. Publications were taken into account in Spanish, English and French, with a publication date of less than or equal to 5 years. Results: The description of treatment, etiology, clinical presentation and diagnosis of Disseminated Intravascular Coagulation was made, with special emphasis on the studies on molecular markers and new therapeutic alternatives. Conclusion: Disseminated intravascular coagulation is a complication which contributes to increase morbidity and mortality, whose early diagnosis and treatment contribute significantly to better clinical evolution.
\end{abstract}

Key words: Disseminated intravascular coagulation; sepsis; anticoagulants. (Source: DeCS, Bireme). 


\section{Introducción}

La coagulación intravascular diseminada (CID) es un síndrome adquirido, secundario a patologías subyacentes, donde la activación localizada de la coagulación y la respuesta inflamatoria generalizada, pueden llevar a daños tisulares y microvasculares donde la isquemia no es fácilmente identificable por métodos clínicos habituales o por mediciones de laboratorio simples, sin embargo se puede manifestar con falla renal, hepática, respiratoria o alteraciones del sistema nervioso central(1-3).

Se conoce que esta entidad clínica se deriva, principalmente de estados infecciosos, desarrollándose en el 25 al $50 \%$ de los pacientes con sepsis de acuerdo con estadísticas a nivel mundial, aunque también se ha reportado su presencia en enfermedad hepática, neoplasias, trauma, y eventos inmunes(4). A pesar de su importancia clínica, existen pocos estudios sobre el tema en los países en vías de desarrollo.

En un estudio realizado durante 7 años en la Clínica Mayo de Minnesota (Estados Unidos), se reportó que a mayor edad se incrementa la incidencia de CID en pacientes críticos, llegando a alcanzar una incidencia de más de 100 casos por 100.000 habitantes por año en hombres mayores de 80 años; respecto a la mortalidad, varía dependiendo de la ubicación geográfica y hospitalaria, la edad del paciente, los criterios diagnósticos y la causa del trastorno hemostático, reportando una mortalidad entre un 22 y $46 \%{ }^{(5,6)}$.

Esta revisión pretende recopilar de la literatura los elementos más destacados de la CID, que permitan conocer y abordar esta complicación desde aspectos fisiopatológicos, clínicos y etiológicos, teniendo en cuenta las investigaciones actuales sobre la enfermedad y los aspectos moleculares que se encuentran en estudio para mejorar la sensibilidad del diagnóstico y la efectividad del tratamiento.

\section{Materiales y métodos}

Se realizó un estudio de revisión de la literatura sobre CID durante el año 2017 por medio de la búsqueda de artículos originales, reportes y series de casos, revisiones sistemáticas y narrativas, en las bases de datos de ciencias de la salud PubMed y ScienceDirect y en el buscador Google Scholar. Se incluyeron artículos disponibles bajo las licencias otorgadas por la Universidad Pontificia Bolivariana. Se seleccionaron en total 80 artículos, de los cuales sólo se incluyeron 51 porque la información no daba respuesta al objetivo planteado, a partir de estos se hizo la descripción del presente texto en el que se desarrolla aspectos relacionados con la Coagulación Extravascular Diseminada como tratamiento, etiología, presentación clínica y diagnóstico. Durante la búsqueda se incluyeron artículos en los idiomas español, inglés y francés. Se favoreció en la búsqueda los artículos con una fecha de publicación menor o igual a 5 años. Las principales palabras de búsqueda en español fueron coagulación intravascular diseminada, sepsis, anticoagulantes. Las principales palabras de búsqueda en inglés fueron disseminated intravascular coagulation, sepsis, anticoagulants.

\section{Fisiopatología}

De acuerdo con el Comité Científico y de Estandarización de la International Society on Thrombosis and Haemostasis (ISTH), la CID se define como un síndrome adquirido donde ocurre una activación intravascular de los sistemas de coagulación sin una localización específica, es decir, de manera sistémica, que puede resultar de diversas causas ${ }^{3,}$ 7. Aunque representa del 9 al 19\% de las admisiones a las Unidades de Cuidados Intensivos (UCI), los estudios sobre la incidencia general de esta complicación son limitados(5).

Aunque su fisiopatología no está completamente esclarecida, la CID se relaciona con el aumento de la liberación de factor tisular (TF) y la disminución de los sistemas fibrinolíticos, existiendo una relación con acúmulos de fibrina y desgaste de los sistemas anticoagulantes endógenos ${ }^{(8,9)}$. 
El endotelio es el órgano más importante en la patogénesis de la CID, ya que es el puente entre la lesión tisular y los componentes plasmáticos; es el sitio donde se encuentran los mecanismos hemostáticos: coagulación, anticoagulación, fibrinólisis y la antifibrinólisis; y es el encuentro de estos mecanismos, junto con las diferentes reacciones de la respuesta inflamatoria, generando daños microvasculares en componentes endoteliales como el glicocálix y otras proteínas intra y extracelulares(3).

El TF, presente en el subendotelio, juega un papel central en el inicio de la coagulación: normalmente no está expuesto hasta que ocurre una lesión vascular; en ausencia de esta, los monocitos e incluso las células cancerígenas, pueden expresarlo y activar la coagulación ${ }^{3}$. En la CID se produce una activación celular que genera presencia de micropartículas procoagulantes en el sistema circulatorio, que posiblemente desequilibra la llamada respuesta inmunotrombótica mediada por los neutrófilos, y que lleva a la liberación de citoquinas inflamatorias como factor de necrosis tumoral alfa $(\mathrm{TNF} \alpha)$ e interleucina $1(2,10)$.

Consecuentemente, los mediadores inflamatorios y procoagulantes llevan a un aumento de la trombina, cuya función es convertir el fibrinógeno en fibrina en la última etapa de la cascada de coagulación, produciendo un aumento del gasto de los sistemas anticoagulantes del cuerpo, disminuyendo los niveles séricos de sustancias anticoagulantes endógenas como la antitrombina y la proteína C; la actividad de la antitrombina tiene un valor predictivo negativo en la CID asociada a sepsis, haciendo considerar su suplementación como alternativa de tratamiento específica para CID(1,11,12). Las proteínas C y S pueden disminuir en pacientes con sepsis en consecuencia a la activación del proceso de coagulación, alteración de la síntesis hepática y degradación por la elastasa de los neutrófilos(3). En la Figura 1 se esquematiza el proceso fisiopatológico de la CID

\section{Etiología}

Sepsis: Se define como una respuesta inflamatoria sistémica, ocasionada por un proceso infeccioso, que involucra daños en el endotelio vascular y genera disfunción orgánica(13). Son múltiples los agentes patógenos que pueden llevar a este estado, siendo la Escherichia coli el más conocido, pero sin excluir agentes poco comunes como Acinetobacter spp. o Arthrobacter spp(14-16). En la sepsis, existe una activación del sistema de coagulación mediada por la generación de trombina, que se logra evidenciar en pruebas de laboratorio como recuento plaquetario, tiempo de protrombina (TP), tiempo parcial de tromboplastina (TPT), dímero D y fibrinógeno(17).

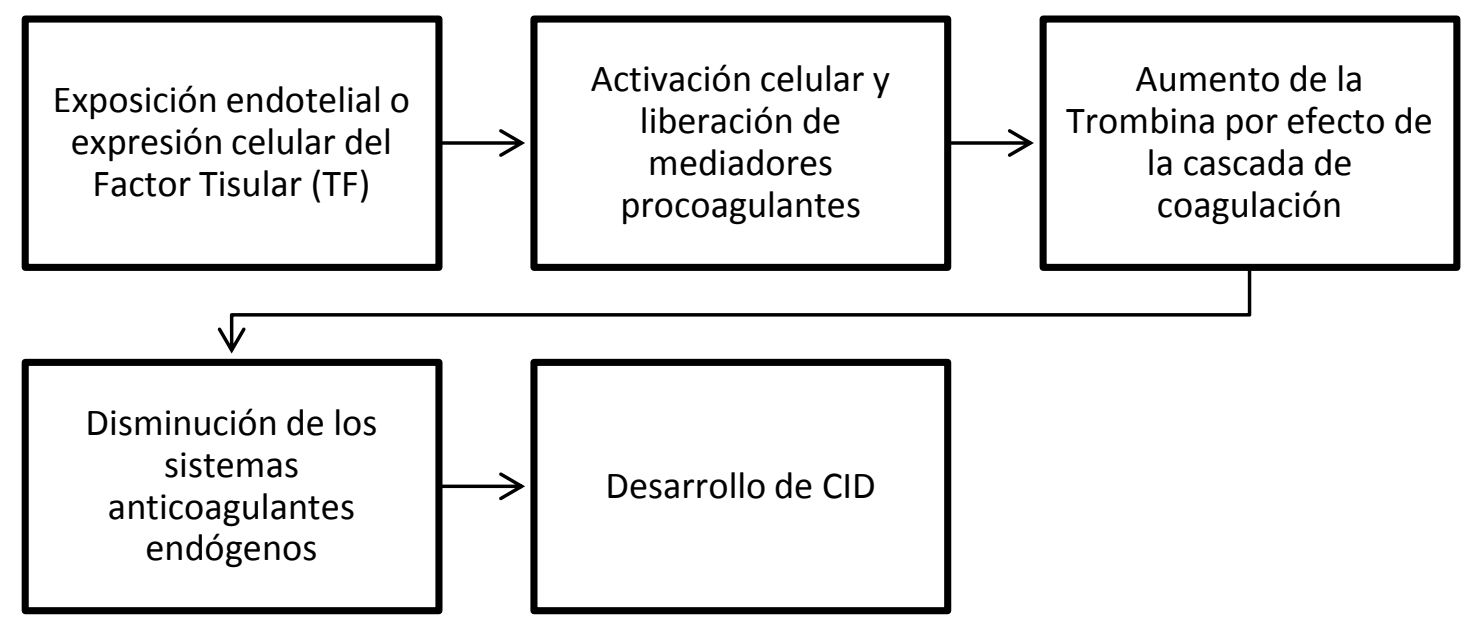

Figura 1. Fisiopatología de la Coagulación Extravascular Diseminada (CID) 
Se ha reportado una prevalencia para la CID del $10,8 \%$ en varias UCI de Colombia; su presentación en el contexto de sepsis es diferente según el tipo de infección, por ejemplo, tiene mayor incidencia en infecciones diferentes a neumonía(18,19). En dos estudios realizados en Colombia, se concluyó que las bacterias GramNegativas son los principales agentes patógenos asociados a sepsis, seguidos por las GramPositivas $(14,20)$. La aparición de CID por agentes menos reportados como Plasmodium vivax hace pensar que puede estar subdiagnosticada(21).

Trauma: En un trauma se presenta una respuesta inflamatoria sistémica con activación de la coagulación por la vía del TF y el factor VIIa, debido a la similitud de su presentación clínica y hallazgos con la coagulopatía aguda de trauma o shock (ACOTS, por sus siglas en inglés) pueden considerarse como una misma entidad $(6,22)$.

Neoplasias: La CID ha sido descrita en el contexto de tumores sólidos, siendo la coagulopatía más frecuente en el cáncer de próstata, además en varios tipos de leucemias, casi de manera universal como complicación de la leucemia mieloide aguda; en cualquier caso, aunque las células endoteliales también están involucradas, se cree que la CID en el contexto de las neoplasias está dada por la expresión aumentada de $\mathrm{TF}$ en las células cancerígenas o en los monocitos o macrófagos, activando así el proceso de la coagulación de manera difusa(23,24).

Embarazo: La CID afecta a 12,5/10.000 embarazos y es la segunda causa de morbilidad materna severa en las admisiones obstétricas(25). Puede resultar de complicaciones no asociadas o asociadas al embarazo entre las primeras se encuentran la sepsis o el trauma y entre las segundas están: hemorragia periparto aguda, desprendimiento placentario, preeclampsia, eclampsia, síndrome de hemólisis, elevación de enzimas hepáticas, trombocitopenia (HELLP), nacimientos muertos retenidos; aborto séptico e infección intrauterina entre otras(26).

La mayoría de las CID obstétricas se deben a hemorragia masiva por lo que el tratamiento de elección inicial es controlar la hemorragia con plasma, plaquetas y crioprecipitado(27). Se asocia con efectos adversos como transfusión masiva de componentes sanguíneos, histerectomía e incluso la muerte por esto es importante realizar un diagnóstico rápido y un tratamiento efectivo(26).

Según el estudio realizado por Jonard et al., en mujeres embarazadas con complicaciones que requirieron ser ingresadas a UCI, llegaron a la conclusión de que los criterios diagnósticos para la CID definidos por la International Society on Thrombosis and Haemostasis (ISTH) no son recomendados para esta población debido a la baja sensibilidad, por lo que otros estudios están evaluando unos criterios que sean más acordes(28).

\section{Cuadro Clínico}

Como principal causa de CID, es necesario saber identificar el cuadro clínico de la sepsis, que consiste en una respuesta anormal ante una infección, con manifestaciones como pirexia, neutrofilia y alteraciones del ritmo cardiaco, acompañados de características concretas dadas por el sitio inflamatorio y el microorganismo desencadenante(29).

La presentación clínica de la CID es variada, se caracteriza por episodios trombóticos, hemorrágicos o mixtos, dependiendo en gran parte de la enfermedad de base del paciente(30). Clínicamente, el componente hemorrágico se presenta como sangrado abundante o espontáneo, incluso en traumatismos leves, adicionalmente pueden encontrarse epistaxis y gingivorragias(31).

El Síndrome de Disfunción Orgánica Múltiple (MODS) surge especialmente en pacientes con alto riesgo de muerte(17). Este síndrome se define como una afectación de dos o más sistemas orgánicos que no estaba presente al momento de ingreso a UCI que puede ser reversible o no(32). En algunos pacientes la presentación puede ser asintomática, siendo los hallazgos de laboratorio los que sugieren CID, aunque el desarrollo de tromboembolismo arterial $o$ venoso $o$ endocarditis no infecciosa deben ser tenidos en cuenta en el cuadro clínico(24). 


\section{Diagnóstico}

Actualmente existe una variedad de criterios diagnósticos para la CID, cuyo uso no está estandarizado y varía según el contexto; estos criterios incluyen los propuestos por la ISTH y la Japanese Association for Acute Medicine (JAAM), los más utilizados hoy en día en el ámbito clínico, así como otras escalas de creación reciente que buscan aumentar la especificidad y la facilidad del diagnóstico(33).

La valoración propuesta por la ISHT, por ejemplo, asigna un puntaje que, de ser mayor o igual a cinco, se considera diagnóstico(34); los cálculos para estas escalas tienden a basarse en pruebas de laboratorio de rutina centradas en medir el consumo de plaquetas o factores de coagulación(35). En la tabla 1 se pueden observar los criterios propuestos por la ISHT con su respectivo puntaje ${ }^{(34)}$. Algunos autores consideran que, no poder diagnosticar la CID en sus etapas más tempranas a partir de los criterios actuales, obligan a que estos sean reemplazados o acompañados por otras pruebas que puedan detectar y clasificar la CID incluso cuando es asintomática(36).

Sin embargo, la necesidad una mayor sensibilidad al momento del diagnóstico ha llevado a la realización de múltiples estudios sobre posibles marcadores moleculares para aplicar a futuro. Un compuesto que ha demostrado ser valioso es la presepsina, un fragmento del cluster de diferenciación 14 (CD14), que da un diagnóstico más preciso al ser medido en sangre, con niveles

Tabla 1. Variables utilizadas para los criterios diagnósticos para Coagulación Intravascular Diseminada de la ISHT

\begin{tabular}{lrrr}
\hline $\begin{array}{l}\text { Variable } \\
\text { evaluada }\end{array}$ & Unidades & $\begin{array}{c}\text { Valores } \\
\text { evaluados }\end{array}$ & Puntaje \\
\hline $\begin{array}{l}\text { Recuento de } \\
\text { plaquetas }\end{array}$ & Células/L & $\begin{array}{r}50 \times 10^{9} \mathrm{a} \\
100 \times 10^{9}\end{array}$ & 0 a 2 \\
$\begin{array}{l}\text { Prolongación } \\
\text { del TP }\end{array}$ & $\mathrm{s}$ & $3 \mathrm{a} 6$ & 0 a 2 \\
$\begin{array}{l}\text { Fibrinógeno } \\
\text { Dímero D }\end{array}$ & $\mathrm{g} / \mathrm{L}$ & 1.0 & 0 a 1 \\
\hline $\begin{array}{l}\text { ISHT: International Society on Thrombosis and Haemostasis } \\
\text { TP: Tiempo de protrombina }\end{array}$ & \\
\end{tabular}

que aumentan proporcionalmente a la severidad del cuadro clínico ${ }^{(37)}$. En conjunto con la proteína $\mathrm{C}$ se ha determinado el criterio Sepsis Induced Disseminated Intravascular Coagulation (SEDIC) aplicable a diagnóstico, clasificación de la severidad y pronóstico(8).

La formación de microtrombos por agregación plaquetaria se asocian con niveles elevados del Factor Inhibidor del Activador del Plasminógeno (PAI-1), medible por Ensayo inmunoabsorbente ligado a enzima (ELISA), y deficiencia de proteasa de escisión del factor de von Willebrand, por lo cual estos compuestos pueden ser usados como marcadores ${ }^{(1,13)}$. También se han considerado el componente C3 del complemento, el Complejo de Ataque de Membrana (MAC) y la Lectina de Unión a Manosa (MBL), que se encontraron elevados en pacientes sépticos con CID, al igual que los complejos monoméricos de fibrina y los productos de degradación de la misma, como el dímero $\mathrm{D}^{(38,39)}$. En cuanto a los complejos DNA-Histona y al DNA de doble cadena (dsDNA), resultantes del proceso de formación de Trampas Extracelulares de Neutrófilos (NETs), estos han demostrado un valor pronóstico sobre la severidad del proceso de coagulación(40).

\section{Complicaciones}

El mayor riesgo de los pacientes con CID asociada a infección son los episodios trombóticos, especialmente venosos $y$ pulmonares, también se pueden presentar eventos arteriales(41). En los casos de sepsis puerperal o episodios sépticos en el embarazo, las complicaciones más importantes son la muerte materna o fetal que, en parte, pueden derivarse de la pérdida de irrigación a los órganos bien sea por oclusión o disminución del volumen sanguíneo(16,42). El MODS, es una de las complicaciones más peligrosas de la CID por su elevada mortalidad(17). La gangrena periférica simétrica, dada por vaso oclusión, se considera una complicación rara, pero debe ser tenida en cuenta por su gravedad, puesto que puede llevar a amputaciones ${ }^{(43)}$. 


\section{Tratamiento actual}

Aunque la resolución del evento desencadenante o enfermedad subyacente suele ser suficiente para la corrección de la CID, el tratamiento directo de los problemas en la hemostasia permite una mejor evolución ${ }^{(44,45)}$. El tratamiento directo aún no está estandarizado, y varía según el país y la fuente que se consulte. En Estados Unidos, el único tratamiento aprobado hasta el año 2012 para la CID asociada a sepsis era la Proteína $C$ activada (APC), con el uso de heparina sólo como profilaxis en pacientes sin sangrado activo $^{41}$. En 2014, se reportó que la APC fue removida del mercado a causa de un estudio donde no demostró ninguna ventaja con respecto a un placebo, aunque en investigaciones previas había logrado reducir la mortalidad(46).

El suplemento de los factores de coagulación y plaquetas también se ha mostrado útil gracias al uso de tecnologías modernas como la tromboelastografía para disminuir las complicaciones asociadas a transfusiones(47). El tratamiento de elección a corto plazo para los pacientes con sangrado como manifestación de CID continúa siendo la terapia con componentes sanguíneos, basada en las necesidades identificadas por el clínico en las pruebas de laboratorio del paciente( ${ }^{(7)}$.

La antitrombina (AT), un inhibidor de la trombina y el factor $\mathrm{X}$, funciona como marcador de laboratorio para CID pues su consumo se asocia con la activación de la coagulación, por lo que su suplemento logra regular la alteración(13). Mientras la ISTH la considera como una alternativa de tratamiento aún en investigación, la Japanese Society of Thrombosis Hemostasis y la Japanese Society of Intensive Care Medicine la recomiendan en CID derivada de sepsis, con algunos estudios demostrando efectividad ${ }^{11}$. A pesar de esto, la Italian Society for Haemostasis and Thrombosis no recomienda el uso de AT ni de trombomodulina recombinante humana (rhTM) en estos casos(41). Al ser un cofactor de la heparina, su actividad se aumenta al combinarse con esta, al igual que en los estados de acidosis(17). En Japón, la AT y la rhTM son la vanguardia como monoterapia del tratamiento anticoagulante en CID, pero, en combinación, se ha demostrado mayor efectividad y seguridad(47).

\section{Futuros tratamientos}

En el estudio realizado por Azuma et al., observaron, que el tratamiento con la inmunoglobulina G4 recombinante anti CD33 en pacientes con leucemia promielocítica aguda (LPA), presentaban CID como principal evento adverso, pero su utilidad fue demostrada en pacientes con LPA de edad avanzada, estos pacientes fueron tratados posteriormente con rhTM para la $\mathrm{CID}^{(48)}$.

La rhTM actúa como anticoagulante por la vía de la proteína $\mathrm{C}$ activada, y está en evaluación para ser aprobada en países diferentes a Japón, aunque, como con la AT, inquieta el riesgo de sangrado ${ }^{(49)}$. Incluso con estas preocupaciones, estudios clínicos han indicado una disminución de la mortalidad con el uso de rhTM, con una supervivencia de $64,1 \%$ y $70,7 \%$ en los grupos estudiados(50). Cuenta con propiedades antiinflamatorias al interferir con la activación del complemento, se administra intravenosa y se elimina por los riñones(44). De hecho, en una investigación realizada en neonatos y comparada con pacientes adultos, se mostró mejoría en las pruebas de laboratorio con disminución en los niveles de productos de degradación de la fibrina $\mathrm{y}$ aumento en el conteo de plaquetas y la actividad de $\mathrm{AT}^{(51)}$.

Tang et al., realizaron un estudio en animales, en el que evidenciaron el papel protector del atractilenolido I que es un componente bioactivo de una planta china, sobre la CID inducida por lipopolisacáridos bacterianos, mejorando la coagulación y suprimiendo la inflamación, con la disminución de la expresión del $\mathrm{TNF} \alpha$, reduciendo el daño de órganos y aumentando la tasa de supervivencia(2).

\section{Pronóstico de los pacientes con CID}

Con una mortalidad intrahospitalaria de hasta el 58\% en Estados Unidos, y el alta como desenlace en sólo el $54 \%$ de los pacientes sobrevivientes, la CID es una complicación con un alto impacto en la sobrevida de los pacientes: el desenlace tiende a ser mejor si la causa subyacente es identificada 
y tratada de manera temprana, lo cual se hace especialmente importante por las limitadas opciones de tratamiento para la $\mathrm{CID}^{(5)}$. Aunque la evidencia es limitada, en los pacientes con cáncer se ha postulado que el aumento fibrinólisis promueve las metástasis, generando un pronóstico pobre(23). Sin embargo, si esta complicación es manejada de manera urgente y agresiva, se puede obtener una respuesta positiva al tratamiento y prolongar la supervivencia(24).

\section{Conclusiones}

El presente artículo de revisión de tema recopiló aspectos relevantes de la CID incluyendo, entre otras cosas, una breve descripción de la fisiopatología, sus principales etiologías, el diagnóstico y el tratamiento. Son diversos factores los que contribuyen al desarrollo de esta complicación y a su pronóstico, desde la patología subyacente hasta factores individuales como la edad, los cuales deben ser tenidos en cuenta para poder reconocer e identificar los casos de CID.

La CID es una complicación que contribuye a aumentar la morbilidad y la mortalidad en los pacientes con enfermedades de base. Su pronto diagnóstico y tratamiento aportan significativamente a una mejor evolución clínica, por lo cual actualmente se investigan nuevos tratamientos, así como marcadores moleculares que amplíen las posibilidades diagnósticas.

La mayor parte de los estudios, así como las guías para el diagnósticos y el tratamiento, son han sido realizados en países desarrollados como Estados Unidos, Japón e Italia, por lo que se hacen necesarios estudios que evalúen la aplicabilidad de esta información en el contexto de países como Colombia, así como estudios que permitan conocer el enfoque actual y la epidemiología de la CID en nuestro medio.

\section{Conflicto de intereses}

Los autores declaran no tener ningún conflicto de intereses.

\section{Referencias}

1. Iba T, Ito T, Maruyama I, Jilma B, Brenner T, Müller MC, et al. Potential diagnostic markers for disseminated intravascular coagulation of sepsis. Blood Rev. 2016;30(2):149-155.

2. Tang XM, Liao ZK, Huang YW, Lin X, Wu LC. Atractylenolide I protects against lipopolysaccharideinduced disseminated intravascular coagulation by anti-inflammatory and anticoagulation effect. Asian Pac J Trop Med. 2017;10(6):582-587.

3. Thachil J. Disseminated intravascular coagulation - new pathophysiological concepts and impact on management. Expert Rev Hematol. 2016;9(8):803-14.

4. Arango M. Coagulación Intravascular Diseminada. Iatreia, 2010; 23(4): 344-353.

5. Singh B, Hanson AC, Alhurani R, Wang S, Herasevich V, Cartin-Ceba $\mathrm{R}$, et al. Trends in the incidence and outcomes of disseminated intravascular coagulation in critically ill patients (2004-2010): a population-based study. Chest. 2013;143(5):1235-1242.

6. Gando S, Levi M, Toh CH. Disseminated intravascular coagulation.Nat. Rev. Dis. Primers. 2016;2:16037.

7. Boral B, Williams D, Boral L. Disseminated Intravascular Coagulation. Am J Clin Pathol 2016;146(6):670-680.

8. Ishikura H, Nishida T, Murai A, Nakamura Y, Irie Y, Tanaka J, et al. New diagnostic strategy for sepsisinduced disseminated intravascular coagulation: a prospective single-center observational study. Critical Care. 2014; 18:R19.

9. Matsumoto $\mathrm{H}$, Yamakawa $\mathrm{K}$, Ogura $\mathrm{H}, \mathrm{Koh} \mathrm{T}$, Matsumoto N, Shimazu T. Enhanced expression of cellspecific surface antigens on endothelial microparticles in sepsis-induced disseminated intravascular coagulation. Shock. 2015;43(5):443-449.

10. Delabranche X, Stiel L, Severac F, Galoisy AC, Mauvieux L, Zobairi F, et al. Evidence of Netosis in Septic ShockInduced Disseminated Intravascular Coagulation. Shock. 2017; 47(3):313-317.

11. Tagami T, Matsui $H$, Fushimi $K$, Yasunaga $H$. Supplemental dose of antithrombin use in disseminated intravascular coagulation patients after abdominal sepsis. Thromb Haemost. 2015; 114(3):537-545.

12. Iba T, Saitoh D, Gando S, Thachil J. The usefulness of antithrombin activity monitoring during antithrombin supplementation in patients with sepsis-associated disseminated intravascular coagulation. Thromb Res. 2015;135(5):897-901.

13. Madoiwa S. Recent advances in disseminated intravascular coagulation: endothelial cells and fibrinolysis in sepsis-induced DIC. J Intensive Care. 2015; 3:8.

14. De La Rosa G, León AL, Jaimes F. Epidemiología y pronóstico de pacientes con infección del torrente sanguíneo en 10 hospitales de Colombia. Rev Chilena Infectol. 2016;33(2):141-149.

15. Baldeo C, Isache C, Baldeo C, Bajwa A. A case of disseminated intravascular coagulation secondary to Acinetobacter lwoffii and Acinetobacter baumannii bacteremia. IDCases. 2015;2(3):70-71. 
16. Shigeta N, Ozaki K, Hori K, Ito K, Nakayama M, Nakahira $\mathrm{K}$, et al. An Arthrobacter spp. Bacteremia Leading to Fetal Death and Maternal Disseminated Intravascular Coagulation. Fetal and Pediatric Pathology 2013; 32:25-31.

17. Liu XL, Wang XZ, Liu XX, Hao D, Jaladat Y, Lu F, et al. Low-dose heparin as treatment for early disseminated intravascular coagulation during sepsis: A prospective clinical study. Exp Ther Med. 2014; 7(3):604-60.

18. Ortíz G, Dueñas C, Rodríguez F, Barrera L, de La Rosa G, Dennis R, et al. Epidemiology of sepsis in Colombian intensive care units. Biomedica. 2014;34(1):40-47.

19. Park JY, Park S, Park SY, Sim YS, Kim JH, Hwang YI, et al. Day 3 versus Day 1 disseminated intravascular coagulation score among sepsis patients: a prospective observational study. Anaesth Intensive Care. 2016;44(1):57-64.

20. Pertuz-Meza Y, Pérez-Quintero C, Pabón-Varela Y. Aspectos epidemiológicos de la sepsis, en unidades de cuidados intensivos Santa Marta, Colombia. Duazary. 2016;13 (2): 126-132.

21. Mohapatra S, Samantaray JC, Arulselvi S, Ghosh A. Disseminated intravascular coagulation following malaria due to Plasmodium vivax: a thromboelastography-based study. Malaria Journal. 2013;12:336.

22. Oshiro, A, Yanagida Y, Gando S, Henzan N, Takahashi I, Makise H. Hemostasis during the early stages of trauma: comparison with disseminated intravascular coagulation. Critical Care, 2014;18(2):R61.

23. Anselmo M, Nobre-de-Jesus G, Madeira J, Victorino R, Meneses-Santos J. Massive Bleeding as the First Clinical Manifestation of Metastatic Prostate Cancer due to Disseminated Intravascular Coagulation with Enhanced Fibrinolysis. Hematology. 2016;2016: 7217915.

24. Feinstein D. Disseminated intravascular coagulation in patients with solid tumors. Oncol J. 2015;29(2):96-102

25. Creanga AA, Berg CJ, Syverson C, Seed K, Bruce FC, Callaghan WM. Pregnancy-related mortality in the United States, 2006-2010. Obstet Gynecol. 2015;125(1):5-12.

26. Erez 0. Disseminated intravascular coagulation in pregnancy - Clinical phenotypes and diagnostic scores. Thromb Res. 2017;151 Suppl 1:S56-S60.

27. Vaught AJ. Critical Care for the Obstetrician and Gynecologist: Obstetric Hemorrhage and Disseminated Intravascular Coagulopathy. Obstet Gynecol Clin North Am. 2016;43(4):611-622.

28. Jonard M, Ducloy-Bouthors AS, Fourrier F. Comparison of Two Diagnostic Scores of Disseminated Intravascular Coagulation in Pregnant Women Admitted to the ICU. PLoS One. 2016;11(11):e0166471.

29. Singer M, Deutschman CS, Seymour CW, Shankar-Hari M, Annane D, Bauer M, et al. TheThird International Consensus Definitions for Sepsis and Septic Shock (Sepsis-3).JAMA. 2016;315(8):801-810.

30. Arslansoyu S, Çakir M, Bahat E, Kaya A, Kerimoglu S, Ökten A. Compartment syndrome, disseminated intravascular coagulation, pneumonia, and acute renal failure due to varicella in a previously healthy child. Scand J Infect Dis. 2014; 46:471-474.
31. Cunnigham G, Nelson D. Síndromes de Coagulación Intravascular Diseminada en Obstetricia. Obstet Gynecol. 2015; 126:999-1011.

32. Ramírez M. Multiple Organ Dysfunction Syndrome. Curr Probl Pediatr Adolesc Health Care. 2013; 43(10): 273277.

33. Ha SO, Park SH, Hong SB, Jang S. Performance Evaluation of Five Different Disseminated Intravascular Coagulation (DIC) Diagnostic Criteria for Predicting Mortality in Patients with Complicated Sepsis. J Korean Med Sci. 2016; 31:1838-1845.

34. Yin Q, Liu B, Chen Y, Zhao Y, Li C. Prognostic value of the International Society on Thrombosis and Haemostasis scoring system for overt disseminated intravascular coagulation in emergency department sepsis. Infection 2014;42:629-637.

35. Levi M. Another step in improving the diagnosis of disseminated intravascular coagulation in sepsis. Critical Care 2013,17:448.

36. Delabranche X, Quenot JP, Lavigne $T$, Mercier E, François B, Severac F, et al. Early Detection of Disseminated Intravascular Coagulation During Septic Shock: A Multicenter Prospective Study. Crit Care Med. 2016;44 (10):e930-e939.

37. Takahashi G, Shibata S, Ishikura H, Miura M, Fukui Y, Inoue $\mathrm{Y}$ et al. and Shigeatsu Endo. Presepsin in the prognosis of infectious diseases and diagnosis of infectious disseminated intravascular coagulation. Eur J Anaesthesiol. 2015;32:199-206.

38. Seki Y, Wada H, Kawasugi K, Okamoto K, Uchiyama T, Kushimoto $\mathrm{S}$, et al. A prospective analysis of disseminated intravascular coagulation in patients with infections. Intern Med. 2013;52(17):1893-1898.

39. Zhao X, Chen YX, Li CS. Predictive value of the complement system for sepsis-induced disseminated intravascular coagulation in septic patients in emergency department. Journal of Critical Care. 2015;30:290-295.

40. Kim JE, Lee N, Gu JY, Yoo HJ, Kim HK. Circulating levels of DNA-histone complex and dsDNA are independent prognostic factors of disseminated intravascular coagulation. Thromb Res. 2015;135(6):1064-9.

41. Lee J, Chul Nam H, Gyoung Kim B, Gyung Kim H, Chan Jung $\mathrm{H}$, Hee Kim J, et al. Renal artery thrombosis secondary to sepsis-induced disseminated intravascular coagulation in acute pyelonephritis. Kidney Res Clin Pract. 2012;31(4):242-245.

42. Khaskheli MN, Baloch S, Sheeba A. Risk factors and complications of puerperal sepsis at a tertiary healthcare centre. Pak J Med Sci. 2013; 29(4):972-976.

43. Sánchez S, Herrera L, Ortiz D, Maya M. Gangrena simétrica periférica en una paciente con choque séptico Septic shock-induced by a symmetrical peripheral gangrene. MULTIMED Revista Médica Granma. 2017;20(5).

44. Vincent JL, Ramesh MK, Ernest D, LaRosa SP, Pachl J, Aikawa N, et al. A randomized, double-blind, placebocontrolled, Phase $2 \mathrm{~b}$ study to evaluate the safety and efficacy of recombinant human soluble thrombomodulin, ART-123, in patients with sepsis and 
suspected disseminated intravascular coagulation. Crit Care Med. 2013;41(9):2069-2079.

45. Nieto JF, Gómez SM, Moncada DC, Serna LM, Hidrón AI.Tratamiento exitoso de linfohistiocitosis hemofagocítica y coagulación intravascular diseminada secundarias a histoplasmosis en un paciente con HIV/sida. Biomédic. 2016;36(1):9-14.

46. Levi M. Diagnosis and treatment of disseminated intravascular coagulation. Int $\mathrm{J}$ Lab Hematol. 2014;36(3):228-236.

47. Crochemore T, Nunes F, Menezes C, Lima L,Zanella PP, Domingos T. Thromboelastometry-guided blood transfusion in septic shock complicated with disseminated intravascular coagulation: a case report. Clin Case Rep. 2017; 5(5):701-706.

48. Azuma Y, Nakaya A, Hotta M, Fujita S, Tsubokura Y, Yoshimura $\mathrm{H}$, et al. Disseminated intravascular coagulation observed following treatment with gemtuzumab ozogamicin for relapsed/refractory acute promyelocytic leukemia. Mol Clin Oncol. 2016;5(1):3134.

49. Ito T, Nagahara A, Osada T, Kato J, Ueyama H, Saito H, et al. Efficacy of recombinant human soluble thrombomodulin in patients with sepsis and disseminated intravascular coagulation in the gastroenterology field. Biomed Rep. 2015; 3(4):457460.

50. Mimuro J, Takahashi H, Kitajima I, Tsuji H, Eguchi Y, Matsushita $\mathrm{T}$, et al. Impact of recombinant soluble thrombomodulin (thrombomodulin alfa) on disseminated intravascular coagulation. Thromb Res. 2013;131(5):436-443.

51. Shirahata A, Mimuro J, Takahashi H, Kitajima I, Tsuji H, Eguchi Y, et al. Recombinant soluble human thrombomodulin (thrombomodulin alfa) in the treatment of neonatal disseminated intravascular coagulation. Eur J Pediatr. 2014;173(3):303-311 